\title{
Biodegradable polymeric microcapsules for selective ultrasound-triggered drug release $\dagger$
}

\author{
Dennis Lensen, ${ }^{* a b}$ Erik C. Gelderblom, ${ }^{c}$ Dennis M. Vriezema, ${ }^{b}$ Philippe Marmottant ${ }^{d}$ Nico Verdonschot, ${ }^{* e f}$ \\ Michel Versluis, ${ }^{c}$ Nico de Jong ${ }^{* c h}$ and Jan C. M. van Hest ${ }^{* a}$
}

\author{
Received 23rd February 2011, Accepted 2nd April 2011 \\ DOI: 10.1039/c1sm05324h
}

\begin{abstract}
A series of hollow biodegradable polymeric microcapsules were prepared, of which their susceptibility to ultrasound was used for triggered release. High speed imaging of the ultrasound experiments showed a strong correlation between the acoustic pressure needed to activate these microcapsules and their shell thickness to diameter ratio. Based on this information a selective triggering of capsules with two different shell thickness to diameter ratios was successfully performed. The capsules were mixed in a single system and were activated independently from each other by a differentiation in acoustic pressure levels. This application is of great interest in the field of drug delivery, since this system allows for localized multiple drug releases in a selective fashion.
\end{abstract}

\section{Introduction}

Diagnostic ultrasound imaging is nowadays routinely performed with ultrasound contrast agents (UCAs). Typically, UCAs are microcapsules with a diameter between 1 and $10 \mu \mathrm{m}$, which are comprised of a gas-filled interior. The microcapsule shell can be composed of a broad variety of molecules, including lipids, proteins, polysaccharides or synthetic polymers. ${ }^{1-5}$ UCAs scatter ultrasound efficiently and they also respond to low energy ultrasound by emitting harmonic frequencies, resulting in contrast enhancement with respect to the surrounding tissue. Air-filled microbubbles are not very practical as contrast agents, since the air dissolves very rapidly in the blood stream and the bubbles are lost from the circulation before the ultrasound examination can be completed. Fluorinated gases combined with a stabilizing shell are therefore commonly used to stabilize the microbubbles for a sufficiently long time; around 5-10 minutes is

${ }^{a}$ Radboud University Nijmegen, Institute for Molecules and Materials, Nijmegen, The Netherlands. E-mail: J.vanHest@science.ru.nl; Fax: +31 24 3653393; Tel: +31242653204

${ }^{b}$ Encapson B.V., Nijmegen, The Netherlands

${ }^{c}$ University of Twente, MIRA Institute of Biomedical Technology and Technical Medicine, Physics of Fluids group, Enschede, The Netherlands ${ }^{d}$ CNRS/Univ. Grenoble 1, Laboratoire Interdisciplinaire de Physique UMR 5588, Grenoble, France

${ }^{e}$ Radboud University Nijmegen, Medical Centre, Orthopaedic Research Laboratory, Nijmegen, The Netherlands

${ }^{f}$ University of Twente, Department Biomechanical Engineering, Enschede, The Netherlands

${ }^{g}$ Erasmus MC, Department of Experimental Echocardiography, Rotterdam, The Netherlands

'Interuniversity Cardiology Institute of The Netherlands, Utrecht, The Netherlands

$\dagger$ Electronic supplementary information (ESI) available: Additional supporting experimental results. See DOI: $10.1039 / \mathrm{clsm}$ 05324h possible. ${ }^{3,6}$ Alternatively, air-filled microbubbles with fluorinated polymer shells have also been used for this purpose. ${ }^{7-9}$

UCAs are generally classified as soft-shelled or as hard-shelled agents. Soft-shelled agents are microbubbles of which the shell is composed of a lipid monolayer with a thickness of 2-3 nm. They undergo volume expansions and contractions that generate an acoustic signal in the far-field of which the non-linear subharmonic or harmonic components give their specific contrastenhancement for medical ultrasound imaging. ${ }^{10-12}$ A drawback of these soft-shelled agents is that upon expansion and contraction of the flexible lipid membrane at elevated pressures, submicron-sized lipid aggregates such as micelles and liposomes are shedded from the microbubble, thereby destroying the UCAs. ${ }^{13}$

The current theoretical models for soft-shelled microbubble triggering using ultrasound incorporate the response of the shell by exploring small amplitude vibration. ${ }^{12,14-17}$ However, for discriminating a contrast agent from the surrounding tissue, a non-linear response of the large amplitude vibration regime is of importance, caused by either buckling ${ }^{18}$ or destruction of the contrast agent. ${ }^{19,20}$ In buckling, a monolayer of lipids shows "compression-only" behavior. ${ }^{21,22}$ Here, only the compression is significant, while no expansion occurs, since these lipid monolayers do not undergo in-plane compression. The bending modulus of the monolayer is very small, so it is energetically more favorable to bend the membrane than to compress it.

Hard-shelled microbubbles have a typical shell thickness in the range of $20-100 \mathrm{~nm}$ and are usually prepared from polymers. They hardly show volume expansions at low acoustic pressure due to the increased damping contribution of the polymer shell and remain intact until a certain pressure threshold is reached. Above this threshold their shell ruptures and the gas core escapes. ${ }^{23}$ It is believed that ultrasound echoes are most likely 
generated only after shell disruption and gas release. However, some hard-shelled microbubbles do generate acoustic signals without losing gas. ${ }^{8}$ These bubbles often indent through a buckling instability, which is a way to conserve their surface area and allow for a change in the volume. By using fluorinated polymers for the microbubble shell, the water intrusion through pores in the shell is very much reduced due to the hydrophobic surface properties of these polymers. ${ }^{7,8,24}$

Recently Marmottant et al. have developed a new theory describing the behavior of hard-shelled microbubbles when undergoing buckling or rupturing using ultrasonic pressure. ${ }^{25}$ Such microbubbles can more easily sustain in-plane compression in contrast to the soft-shelled microbubbles, thus allowing for stabilization against dissolution of the gas inside. This, however, holds for limited compressions as these microbubbles also undergo buckling if the compressive constraint is large enough. They also compared this theoretical study with the experimental results of Bouakaz et al. obtained earlier, in which the contrast agent PB127 (Point Biomedical) was used. ${ }^{19}$

Ultrasound-triggered drug release has attracted increasing interest, since the release trigger can be applied locally. ${ }^{1,5,26}$ Böhmer et al. have recently shown that polymeric PLLA capsules with different shell thicknesses can be triggered both in vitro and in vivo, although using different ultrasonic pressures. ${ }^{26}$ They incorporated a model drug, Evans blue, in liposomes and polymeric capsules with different shell thicknesses and triggered them in a gel using high pressures. By comparing the destruction zone of the capsules as a function of the ultrasonic pressure, they observed that a thicker shell required a higher ultrasonic pressure. They varied the ultrasonic pressure in rather big steps of $1 \mathrm{MPa}$. At higher pressures hard-shelled capsules were destroyed with dramatic changes in the gas volume. ${ }^{8,19,23}$ Although this leads to a loss of US properties after breakage, it also opens up the possibility of using UCAs as drug delivery vehicles, since the capsule content is efficiently released. Furthermore, by applying both low and high US pressure, UCA-based drug delivery vehicles can be used for monitoring and release, respectively, which substantially improves therapeutic treatment. A next step forward would be realized if UCA drug delivery vehicles could be employed in selective drug release. This could be achieved if a mixture of microcapsules is used which releases its encapsulated drugs at different ultrasound parameters, such as pressure, pulse repetition frequency or pulse length.

In this paper we describe in detail the ultrasonic pressure needed to trigger a series of biodegradable polymeric microcapsules with adjustable sensitivity toward ultrasound. Tuning of the shell thickness of a capsule made it possible to selectively trigger capsules using diagnostic ultrasound at a mechanical index of 1.2, which was well below the maximum mechanical inde $\mathrm{x}^{27}$ of 1.9 as regulated by the FDA. ${ }^{28}$ Also a strong correlation was found between the activation pressure of the ultrasound, the pressure at which a capsule shell buckles or ruptures, and the shell thickness of similar sized capsules. These results were in perfect agreement with the theoretical model of Marmottant et al. for hard-shelled microcapsules. Furthermore, selective triggering of microcapsules in a single system having different shell thicknesses was proven, which is a promising step toward more controlled drug delivery applications.

\section{Experimental}

\section{Materials}

L-Lactide, poly(vinyl alcohol) (PVA, $M_{\mathrm{w}} 9-10 \mathrm{kDa}, 80 \%$ hydrolyzed), methoxy-poly(ethylene glycol) (PEG, $\left.M_{\mathrm{w}}=3350\right)$, tetrahydrofuran (THF) and stannous(II) octanoate $\left(\mathrm{Sn}(\mathrm{Oct})_{2}\right)$ were purchased from Sigma-Aldrich (Schnelldorf, Germany). Decane was purchased from Fluka (Schnelldorf, Germany). 2,2,3,3,4,4,5,5,6,6,7,7,8,8,8-pentadecafluoro-1-octanol (PFO) was purchased from ABCR (Karlsruhe, Germany). Methanol was purchased from Baker (Griesheim, Germany). Dichloromethane was distilled over $\mathrm{CaCl}_{2}$ and toluene was distilled over $\mathrm{Na}$ /benzophenone. All other chemicals were used as received.

\section{Equipment}

${ }^{1} \mathrm{H}$ NMR spectra were recorded on a Varian $400 \mathrm{MHz}$ at room temperature and with $\mathrm{CDCl}_{3}$ as a solvent containing tetramethylsilane as the internal standard.

Molecular weights of the polymers were measured using size exclusion chromatography (SEC) on a Shimadzu system equipped with a guard column and a PL gel $5 \mu \mathrm{m}$ mixed D column (Polymer Laboratories) with a differential refractive index detector, using THF as an eluent at $1 \mathrm{~mL} \mathrm{~min}^{-1}$ and $T=35^{\circ} \mathrm{C}$. Polystyrene standards in the range of 580 to $377400 \mathrm{~g} \mathrm{~mol}^{-1}$ (Polymer Laboratories) were used to calibrate the SEC.

Differential Scanning Calorimetry (DSC) measurements were performed on a Perkin Elmer Pyris Diamond DSC, equipped with a Perkin Elmer Intracooler 2P. Samples were prepared in an aluminium cup with less than $1 \mathrm{mg}$ of sample. Empty aluminium cups were used as a reference. All samples were measured with heating rates of $5^{\circ} \mathrm{C}$ minute ${ }^{-1}$.

Cryo-SEM was performed on a JEOL JSM-6330F electron microscope. All samples were sputtered with a layer of $\mathrm{Pd} / \mathrm{Au}$.

Samples used for lyophilization were rapidly frozen in liquid nitrogen. Lyophilization was performed with an Ilshin FD 8515 with a condenser temperature of $-92{ }^{\circ} \mathrm{C}$ at $5 \mathrm{mT}$ Torr for at least 24 hours.

\section{Synthesis of PFO-PLLA}

Poly(L-lactic acid) terminated by PFO was synthesized according to Lee et al. ${ }^{24}$ PFO (1.02 g, $\left.2.55 \mathrm{mmol}\right)$ and L-lactide (6.61 g, 0.46 $\mathrm{mol})$ were suspended in toluene $(50 \mathrm{~mL})$ under an argon atmosphere, while stirring. After 5 minutes $\mathrm{Sn}(\mathrm{Oct})_{2}(10 \mathrm{mg}, 26 \mu \mathrm{mol})$, dissolved in toluene $(1 \mathrm{~mL})$, was added to the suspension and the mixture was heated to $130{ }^{\circ} \mathrm{C}$ for 18 hours. The mixture was allowed to cool to room temperature and evaporated to dryness. The resulting white polymer was dissolved in a minimal amount of dichloromethane and precipitated in methanol. The precipitate was filtered off and was purified once more by the procedure described above. The precipitate was dried under high vacuum for 24 hours to yield a white solid $(6.30 \mathrm{~g}$, yield: $73 \%)$. The ${ }^{1} \mathrm{H}$ NMR spectrum of the resulting polymer was similar as the polymer described in the literature. ${ }^{7}$

Molecular weight determination: NMR: $M_{\mathrm{n}}=3395 \mathrm{~g} \mathrm{~mol}^{-1}$, GPC: $M_{\mathrm{n}}=6233 \mathrm{~g} \mathrm{~mol}^{-1}, M_{\mathrm{w}}=8583 \mathrm{~g} \mathrm{~mol}^{-1}, \mathrm{DPI}=1.38$. 


\section{General procedure for preparing PFO-PLLA microcapsules}

PFO-PLLA was weighed and dissolved in dichloromethane $(2.0 \mathrm{~g})$ and to this decane $(0.76 \mathrm{~g})$ was added. This solution was added slowly to a solution of PVA in water $(1 \mathrm{wt} \%, 15 \mathrm{~mL})$ stirred at $10000 \mathrm{rpm}$ using an ultraturrax. The formed emulsion was stirred for an additional 5 minutes and then transferred to a beaker containing PVA in water $(1 \mathrm{wt} \%, 40 \mathrm{~mL})$ and stirred for 24 hours using a magnetic stirring bar to allow the dichloromethane to evaporate. The dispersion was centrifuged at 3000 $\mathrm{rpm}(605 \mathrm{~g})$ for 10 minutes, followed by isolation of the top layer of floating capsules. The capsules were redispersed in a PEG solution $\left(5 \mathrm{wt}^{\mathrm{O}} \%\right.$ ) and centrifuged twice more at $3000 \mathrm{rpm}(605 \mathrm{~g})$, each time redispersing the floating capsules. Finally the floating capsules were isolated, redispersed in a minimal amount of PEG solution and lyophilized to remove the decane from the lumen. These gas-filled capsules could be stored for more than 1 year, while remaining ultrasonically active in solution.

Fluorescent PFO-PLLA capsules were prepared identical to the procedure described above, only Nile Red (1 mg) was added to the polymer solution.

\section{Ultrasound measurements}

Microcapsules were dispersed in double distilled water (dd water, $R=18.2 \mathrm{M} \Omega$ ) and injected in an Opticell. This cell was placed under water and the microcapsules were measured by a setup described by Overvelde $e t a l .{ }^{29}$ The capsules were insonified using a 1.0 MHz single element transducer (Precision Acoustics Ltd) transmitting 16-cycle-sine wave bursts, with pressures between 50 and $1200 \mathrm{kPa}(\mathrm{MI}=0.05-1.2)$, as verified with a calibrated $0.2 \mathrm{~mm}$ PVDF needle hydrophone (Precision Acoustics Ltd). Images of insonified microcapsules were recorded using a $60 \times$ water-immersed objective and $2 \times$ magnifier, and recorded in six sequences of 128 image frames at a frame rate near 5 million frames per second using the Brandaris 128 ultrahigh-speed camera system. ${ }^{29}$ Images were processed using custom-designed software written in MATLAB (Mathworks).

For the selective ultrasound measurement an identical ultrasound setup was used as described above. The capsules were insonified using a $1.0 \mathrm{MHz}$ single element transducer (C302; Panametrics-NDT; Olympus-NDT) transmitting 16-cycle-sine wave bursts, with pressures between 50 and $1200 \mathrm{kPa}(\mathrm{MI}=$ 0.05-1.2), as verified with a calibrated $1.0 \mathrm{~mm}$ PVDF needle hydrophone (Precision Acoustics Ltd). The PFO-PLLA capsules with Nile Red were visualized using an excitation wave length of $550 \mathrm{~nm}$ and emission snapshots were recorded at $580 \mathrm{~nm}$. Overlay images of bright-field and fluorescent snapshots of insonified microcapsules were recorded using a $40 \times$ waterimmersed objective and $2 \times$ magnifier using custom-designed software written in MATLAB (Mathworks).

\section{Cryo-SEM measurements of PFO-PLLA capsules}

Lyophilized PFO-PLLA capsules were dispersed in dd water and manually shaken in an eppendorf tube. The dispersion was centrifuged at $3000 \mathrm{rpm}(605 \mathrm{~g})$ and the supernatant was taken out using a syringe fitted with a needle. The capsules were redispersed in dd water and the procedure was performed two more times. Finally the capsules were dispersed in dd water and rapidly frozen in liquid nitrogen.

\section{Results and discussion}

For the preparation of ultrasound responsive microcapsules with different shell thicknesses, an emulsion-evaporation technique was employed. ${ }^{30}$ Here, an oil-in-water emulsion was used, in which the oil phase consisted of a mixture of the shell-forming polymer, in this case perfluoroctanol-poly(lactic acid) (PFOPLLA) ${ }^{24}$ a volatile solvent, dichloromethane, and a non-volatile non-solvent, decane. As the dichloromethane started to evaporate small polymer-rich droplets were formed by phase separation within the emulsion droplets. These polymer-rich droplets migrated to the interface and engulfed under the right conditions the original droplet. Further removal of the volatile solvent resulted in more polymer precipitate which migrated to the shell, thus forming core-shell microcapsules. By using different initial concentrations of polymer in the organic phase and by maintaining the same mechanical energy for droplet formation, four different capsules were prepared with increasing shell thickness, see Table 1 .

In order to obtain hollow biodegradable microcapsules, decane was removed from the core using lyophilization. No deformations of the capsules were observed before and after lyophilization as was presented in a previous study. ${ }^{31}$ The removal of decane from the core of these capsules was confirmed by DSC measurements ( $\mathrm{S} 1$ in the $\mathrm{ESI} \dagger$ ), as it was observed that no melting peak for decane was found at $-28{ }^{\circ} \mathrm{C}$ after freeze drying for all four types of capsules.

By using cryo-SEM it was also possible to determine the capsule diameters and their shell thicknesses (Table 1) as depicted in Fig. 1. Furthermore, the dispersities of the capsule sizes $(5.3 \mu \mathrm{m} \pm 2.3 \mu \mathrm{m})$ have also been determined from SEM measurements and are depicted in the ESI $\uparrow(\mathrm{S} 2)$.

From Fig. 1B it is observed that the ratio between the shell thickness and the diameter shows a gradual increase upon increasing the polymer mass, which demonstrates that there is good control over the desired shell thickness. However, there is a slight deviation of the ratio shell thickness to diameter, since the thickness of the shell is not completely homogeneous. Furthermore, as expected, larger capsules have thicker shells as a result of a larger initial droplet size, since more decane in the core will result in more polymer ending up in the shell.

Listed in Table 1 are the calculated shell thicknesses for these capsules by applying eqn (S1) (denoted in the ESI $\dagger$ ). The calculated shell thicknesses were slightly lower than the measured values, which can be partly explained by the fact that the exact capsule radius was difficult to estimate from cryo-SEM, since the capsules were not fully exposed from the ice. Moreover, the calculation assumes ideal mixing conditions of the decane and the polymer solution and that all polymer material ends up in a microcapsule. This, however, may not have been achieved during microcapsule preparation and may have therefore led to an overestimation of the shell thickness. In order to investigate the effect of the ratio of the shell thickness to the capsule size on the response to ultrasound pressure, the capsule dynamics were studied optically using the Brandaris 128 ultra high-speed camera during insonification of the capsules. ${ }^{32}$ The pressure pulse 
Table 1 Overview of the properties of PFO-PLLA microcapsules prepared using an emulsion-evaporation technique with different polymer concentrations

\begin{tabular}{|c|c|c|c|c|c|}
\hline Capsule & $\begin{array}{l}\text { Polymer } \\
\operatorname{mass}^{a} / \mathrm{mg}\end{array}$ & $\begin{array}{l}\text { Calculated shell } \\
\text { thickness } / \mathrm{nm}\end{array}$ & $\begin{array}{l}\text { Measured shell } \\
\text { thickness } / \mathrm{nm}\end{array}$ & $\begin{array}{l}\text { Measured capsule } \\
\text { diameter }{ }^{c} / \mu \mathrm{m}\end{array}$ & $\begin{array}{l}\text { Ratio between shell } \\
\text { thickness and diameter }\left(10^{-3}\right)\end{array}$ \\
\hline 1 & 100 & 43 & $65 \pm 2$ & $3.54 \pm 0.07$ & $18 \pm 0.5$ \\
\hline 4 & 500 & 137 & $158 \pm 6$ & $2.67 \pm 0.11$ & $59 \pm 4.3$ \\
\hline
\end{tabular}

${ }^{a}$ All polymers were dissolved in $1.5 \mathrm{~mL}$ dichloromethane and $1.0 \mathrm{~mL}$ decane. The emulsion was prepared by stirring at $10000 \mathrm{rpm}$ using an ultraturrax. ${ }^{b}$ See ESI $\dagger$ for the calculation. ${ }^{c}$ Average sizes from ten measurements obtained from cryo-SEM studies.
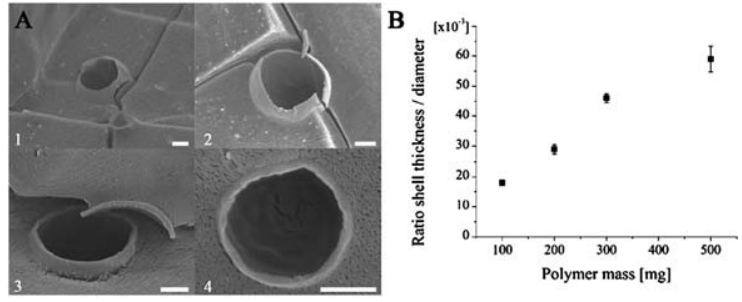

Fig. 1 (A) Scanning electron micrographs of PFO-PLLA microcapsules 1-4 from Table 1 with different shell thicknesses. Scale bars represent 1 $\mu \mathrm{m}$. (B) Plot of the polymer mass versus the ratio of shell thickness to diameter.

that was applied to the microcapsules during ultrasound treatment is depicted in Fig. 2A. The Brandaris camera was operated at a frame rate of 5 million frames per second (Mfps), thus allowing for a highly detailed insight in the behavior of the microcapsules during the applied pressure wave.

At low acoustic pressures (50-250 $\mathrm{kPa}$ ) no capsules were disrupted. However, the capsules with the thinnest shell (entry 1 in Table 1) showed buckling behavior at an acoustic pressure of $250 \mathrm{kPa}$ as shown in Fig. 2B.

Here, the capsule's expansion and contraction correspond to the pressure at different times as indicated in Fig. 2A.

By increasing the acoustic pressure by 40 percent to $350 \mathrm{kPa}$, capsule 1 continued to buckle as depicted in Fig. 2B. However, above a certain acoustic pressure threshold, the shell yielded, resulting in rupture of the shell at its thinnest point and release of the gas content as depicted in Fig. 3(1a-e). The capsules with a higher shell thickness/diameter ratio did not show buckling behavior, apparently due to a shell thickness of these capsules that was too high to allow indentation. All capsules (1-4 from

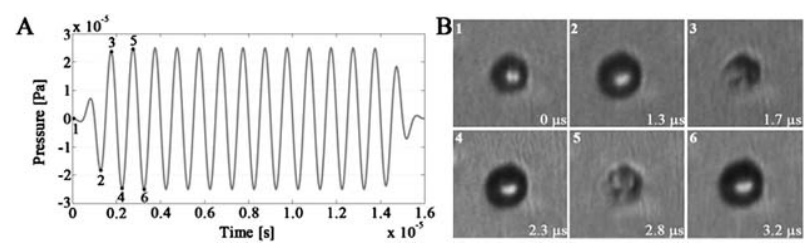

Fig. 2 (A) Schematic representation of the sine wave of the applied ultrasound pressure. (B) Buckling behavior of a thin-shelled polymer capsule (entry 1 in Table 1) at $250 \mathrm{kPa}$. Numbers $1-6$ correspond to the applied pressure as depicted in (A).
Table 1) responded by rupturing when the acoustic pressure was sufficiently high.

From Fig. 3 it can be observed that the release of the gas was more violent when the capsules had a thicker shell/diameter ratio. The capsules with higher ratios could withstand the expansion of the gas when insonified. When even higher acoustic pressures were used to trigger the capsules, the gas in the capsules expanded further, giving rise to a stronger, more impulsive gas release. The data points of the ratio of the calculated shell thickness and diameter of the capsules were plotted against the acoustic pressure of the ultrasound as depicted in Fig. 4.

It shows that the ratio of shell thickness to diameter of the capsules correlates strongly with the ultrasound pressure needed to disrupt the capsules. A buckling behavior was only observed for capsules with low shell/diameter ratios and at low ultrasound pressure. For the capsules with the larger shell thickness/diameter ratio this behavior was no longer observed and the capsules only responded by instant rupturing when the ultrasound pressure was above the release threshold.

This behavior corresponds nicely with the recent theory of Marmottant et al. ${ }^{25}$ where they predicted the rupture and buckling threshold for varying bubble radii. When the shell thickness to bubble diameter ratio is varied, the pressure for

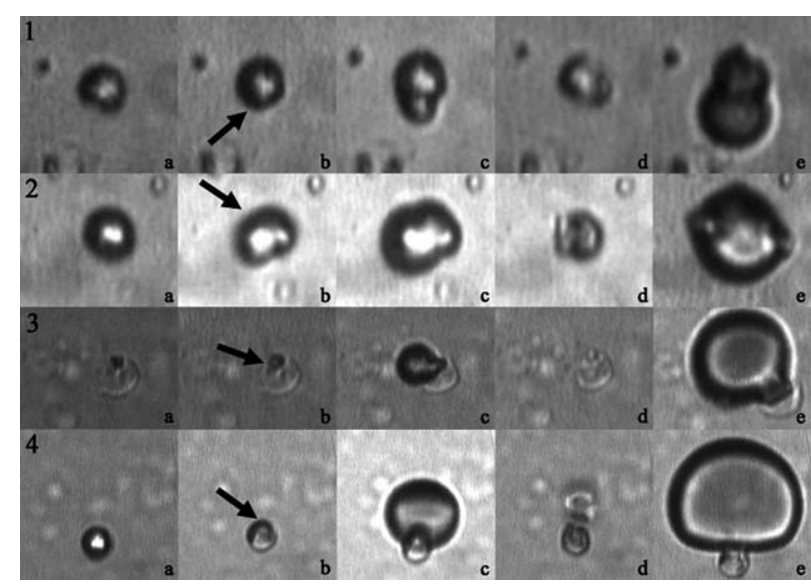

Fig. 3 Rupture of the capsules 1-4 using ultrasound at high acoustic pressure. (a) Capsule just before rupture. (b) Shell of the capsule ruptures (arrow). (c) Gas escapes from the core of the capsule. (d) Contraction of the capsule, when a positive pressure is applied. (e) Second expansion of the gas and full rupture of the capsule, when maximal pressure is applied. Images are snapshots of movies recorded by the Brandaris camera (see ESI $\dagger$ for ultra high-speed movies of the breakup of different capsules). 


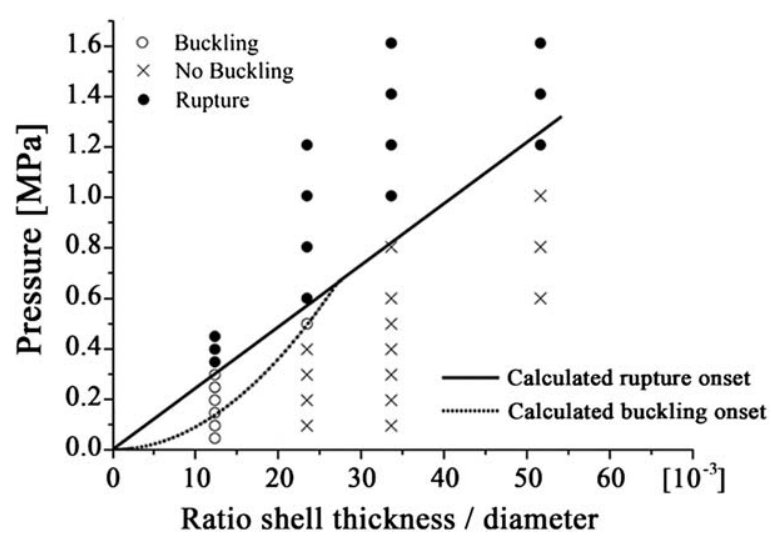

Fig. 4 Plot of the ratio of capsule shell to capsule diameter against the acoustic pressure, together with the theoretical prediction for the onset of buckling and rupturing. The following parameters were used: a Young's modulus of $E=225 \mathrm{MPa}$, and a critical elongation for rupture of $e_{\max }=$ $1.8 \%{ }^{25}$ Symbols in the graph represent the measured data points. The errors for the ratio shell thickness/diameter were calculated from the diameter of the capsules as observed in the high speed recordings and are respectively $12 \pm 1,23 \pm 2,33 \pm 3$ and $51 \pm 4.5$.

buckling onset is predicted to vary as $P_{\text {buckling }}=E(d / R)^{2}$, with $E$ the elastic Young's modulus, $d$ the shell thickness and $R$ the bubble radius. The rupture is achieved when the shell material is elongated more than a critical elongation $e_{\max }$, and occurs at a pressure $P_{\text {buckling }}=3 E(d / R) e_{\max }$. The experimental data were compared with the theory of Marmottant et al. as shown in Fig. $4 .^{25}$ They also stated that above a certain shell thickness/ diameter ratio, no buckling will be observed and the bubbles will only rupture while releasing their gas content, which is in perfect agreement with our observations.

The good control over the ratio of shell thickness to diameter of these capsules, as well as their predictable behavior toward ultrasound pressure, makes these capsules ideal as selective drug delivery vehicles. In order to demonstrate this, two different capsules were mixed with markedly different ratios (capsules 1 and 4 in Table 1). In order to distinguish between these capsules, the thin-shelled capsules were loaded with the dye Nile Red, which was visualized using fluorescence microscopy. An overlay image of the fluorescence microscope image and the bright-field microscope image marks the location of the different capsules as can be seen in Fig. 5A.

By first applying a low pressure $(400 \mathrm{kPa})$, the thin shelled capsules were activated as depicted in Fig. 5B. The wall of these capsules was disrupted allowing water to penetrate into the

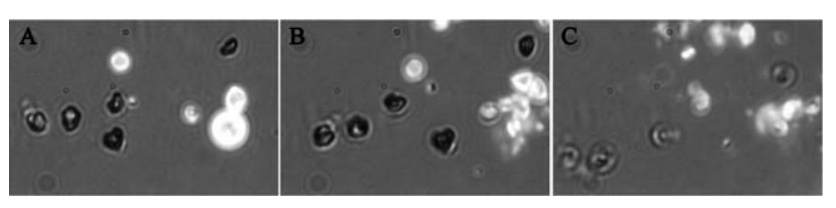

Fig. 5 Overlay microscope images of mixed microcapsules having different ratios of shell thickness to diameter, triggered by ultrasound. (A) Image of the microcapsules when no pressure was applied. (B) Image of the microcapsules when $400 \mathrm{kPa}$ of pressure was applied. (C) Image of the microcapsules when 1.2 MPa was applied. capsules. As a consequence the microcapsules sank and moved out of focus of the microscope. The larger capsule showed a more violent disruption and displacement than the smaller ones, which is due to the bigger volume of gas present in this capsule. From this image it was observed that all the thinner shelled capsules with the same ratio of shell thickness/diameter were triggered. When applying a second pressure pulse of $1.2 \mathrm{MPa}$, also the thicker shelled microcapsules were disrupted (Fig. 5C). It can be clearly seen that the gas, which was entrapped in these capsules had escaped, while the shells of the capsules remained. This demonstrates that capsules with different shell thicknesses can be triggered independently from each other using different ultrasonic pressures, thereby releasing their contents.

\section{Conclusions}

In this paper we have shown the preparation of a series of microcapsules with an adjustable shell thickness to diameter ratio. By controlling the ultrasound parameters we have demonstrated with high speed imaging that different acoustic pressures were needed to disrupt the capsules with varying shell thickness to diameter ratios. Also, the ultrasound behavior of these capsules was proven to be in excellent agreement with the recently developed theory of Marmottant et al. Additionally, we have also shown that capsules with different shell thicknesses to diameter ratios can be triggered independently from each other in a single system, giving the possibility to release drugs in a stepwise or selective manner. Since the US pressures applied were well below the maximum Mechanical Index allowed for diagnostic imaging, this implies that these capsules can be used to deliver drugs without safety hazards.

\section{Acknowledgements}

The authors would like to acknowledge Geert-Jan Jansen for his assistance with the cryo-SEM experiments. This work was supported by the European Commission under no. NMP-2004SME-3.4.4.6.

\section{References}

1 M. R. Bohmer, A. L. Klibanov, K. Tiemann, C. S. Hall, H. Gruell and O. C. Steinbach, Eur. J. Radiol., 2009, 70, 242-253.

2 I. Lentacker, S. C. De Smedt and N. N. Sanders, Soft Matter, 2009, 5, 2161-2170.

3 S. Tinkov, R. Bekeredjian, G. Winter and C. Coester, J. Pharm. Sci., 2009, 98, 1935-1961.

4 A. L. Klibanov, Invest. Radiol., 2006, 41, 354-362.

5 V. Sboros, Adv. Drug Delivery Rev., 2008, 60, 1117-1136.

6 E. G. Schutt, D. H. Klein, R. M. Mattrey and J. G. Riess, Angew. Chem., Int. Ed., 2003, 42, 3218-3235.

7 M. R. Bohmer, R. Schroeders, J. A. M. Steenbakkers, S. de Winter, P. A. Duineveld, J. Lub, W. P. M. Nijssen, J. A. Pikkemaat and H. R. Stapert, Colloids Surf., A, 2006, 289, 96-104.

8 K. Kooiman, M. R. Bohmer, M. Emmer, H. J. Vos, C. Chlon, W. T. Shi, C. S. Hall, S. de Winter, K. Schroen, M. Versluis, N. de Jong and A. van Wamel, J. Controlled Release, 2009, 133, 109-118.

9 C. Chlon, C. Guedon, B. Verhaagen, W. T. Shi, C. S. Hall, J. Lub and M. R. Bohmer, Biomacromolecules, 2009, 10, 1025-1031.

10 M. Emmer, A. Van Wamel, D. E. Goertz and N. De Jong, Ultrasound Med. Biol., 2007, 33, 941-949.

11 J. R. Lindner, Nat. Rev. Drug Discovery, 2004, 3, 527-532.

12 S. M. van der Meer, B. Dollet, M. M. Voormolen, C. T. Chin, A. Bouakaz, N. de Jong, M. Versluis and D. Lohse, J. Acoust. Soc. Am., 2007, 121, 648-656. 
13 J. E. Chomas, P. A. Dayton, D. May, J. Allen, A. Klibanov and K. Ferrara, Appl. Phys. Lett., 2000, 77, 1056-1058.

14 C. C. Church, J. Acoust. Soc. Am., 1995, 97, 1510-1521.

15 A. A. Doinikov and P. A. Dayton, J. Acoust. Soc. Am., 2007, 121, 3331-3340.

16 A. A. Doinikov, J. F. Haac and P. A. Dayton, Ultrasonics, 2009, 49, 269-275.

17 K. Sarkar, W. T. Shi, D. Chatterjee and F. Forsberg, J. Acoust. Soc. Am., 2005, 118, 539-550.

18 E. Stride and N. Saffari, Ultrasound Med. Biol., 2003, 29, 563-573.

19 A. Bouakaz, M. Versluis and N. de Jong, Ultrasound Med. Biol., 2005, 31, 391-399.

20 C. K. Yeh and S. Y. Su, Ultrasound Med. Biol., 2008, 34, 1281-1291.

21 N. De Jong, M. Emmer, C. T. Chin, A. Bouakaz, F. Mastik, D. Lohse and M. Versluis, Ultrasound Med. Biol., 2007, 33, 653-656.

22 P. Marmottant, S. van der Meer, M. Emmer, M. Versluis, N. de Jong, S. Hilgenfeldt and D. Lohse, J. Acoust. Soc. Am., 2005, 118, 3499 3505 .

23 S. H. Bloch, M. Wan, P. A. Dayton and K. W. Ferrara, Appl. Phys. Lett., 2004, 84, 631-633.
24 W. K. Lee, I. Losito, J. A. Gardella and W. L. Hicks, Macromolecules, 2001, 34, 3000-3006.

25 P. Marmottant, A. Bouakaz, N. De Jong and C. Quilliet, J. Acoust. Soc. Am., 2011, 129, 1231-1239.

26 M. R. Böhmer, C. H. T. Chlon, B. I. Raju, C. T. Chin, T. Shevchenko and A. L. Klibanov, J. Controlled Release, 2010, 148, 18-24.

27 R. E. Apfel and C. K. Holland, Ultrasound Med. Biol., 1991, 17, 179185.

28 FDA Device Regulation and Guidance, http://www.fda.gov/ downloads/MedicalDevices/DeviceRegulationandGuidance/ GuidanceDocuments/UCM070911.pdf

29 M. Overvelde, V. Garbin, J. Sijl, B. Dollet, N. De Jong, D. Lohse and M. Versluis, Ultrasound Med. Biol., 2010, 36, 2080-2092.

30 D. Lensen, D. M. Vriezema and J. C. M. van Hest, Macromol. Biosci., 2008, 8, 991-1005.

31 D. Lensen, K. van Breukelen, D. M. Vriezema and J. C. M. van Hest, Macromol. Biosci., 2010, 10, 475-480.

32 C. T. Chin, C. Lancee, J. Borsboom, F. Mastik, M. E. Frijlink, N. de Jong, M. Versluis and D. Lohse, Rev. Sci. Instrum., 2003, 74, 50265034 